\title{
GASTOS E INGRESOS EN LA ACTIVIDAD CAPRINA EXTENSIVA EN MÉXICO'
}

\author{
Samuel Rebollar-Rebollar ${ }^{*}$, Juvencio Hernández-Martínez ${ }^{2}$, Rolando Rojo-Rubio², Eugenio Guzmán-Soria ${ }^{2}$
}

\section{RESUMEN}

Gastos e ingresos en la actividad caprina extensiva en México. El objetivo de este trabajo fue determinar costos de producción y rentabilidad de caprinos en un sistema de producción extensivo. La investigación se realizó durante los meses de enero-junio de 2011 en las localidades: Monte de Dios y San Lucas del Maíz, municipio de Tejupilco, Estado de México; la información provino de una encuesta aplicada a 37 productores. Los costos de producción consideraron solamente conceptos monetarios desembolsados efectivamente por productores; existieron rubros que no se incluyeron, ya que estos los incluyó la economía familiar o cuyos costos de oportunidad fueron iguales a cero; u otros que se omitieron, debido a que no se utilizaron. El costo de producción por cabeza estimado fue US\$8,7, de éste, el costo variable y fijo fue US\$ 3,8 y US\$ 4,9. El componente principal del costo variable fue el concepto de desparasitantes y el fijo el mantenimiento de corrales. El ingreso medio por ventas consideró un peso de $35 \mathrm{~kg}$ y un precio por kilogramo de dos dólares; con lo que el ingreso total por animal fue US\$ 71,2. Con ello, la ganancia neta se ubicó en US\$ 62,5; en tanto que la relación beneficio-costo fue $8: 1$. El costo de producción de caprinos, bajo este sistema de producción, fue menor que la tercera parte del ingreso por venta, debido a la poca magnitud de gastos de producción; por lo que la ganancia por unidad vendida fue siete veces superior al costo de producción.

\begin{abstract}
Income and expenses in the goat extensive activity in Mexico. The objective of this work was to determine production costs and profitability of goats in extensive production systems. The research was carried out during JanuaryJune 2011 in the localities Monte de Dios and San Lucas del Maíz, Tejupilco municipality, State of Mexico. The information came from a survey of 37 producers. Production costs considered money only actually paid by producers, there were items that were not included, since they were absorbed by the family economy, or were opportunity costs equal to zero, or omitted because they were not used in practice. The production cost per head was US\$ 8.7; of this, variable and fixed costs were US\$ 3.8 and US\$ 4.9. The main variable cost was parasiiite control and tha main fixed cost was maintenace of stabullating installations. The average revenue from sales considered a weight of $35 \mathrm{~kg}$ and a price of two dollars per kilogram, bringing the total income to US\$ 71.2 per animal. With this, the net profit was US\$ 62.5 , while the benefit-cost ratio was 8.1 . The cost of producing goats under this system of production was less than a third of revenues from sales, due to the relatively low production costs, so that profit per unit sold was seven times the cost of production.
\end{abstract}

Keywords: Fixed cost, variable cost, net profit, goats, inputs.

Palabras clave: Costo fijo, costo variable, ganancia neta, caprinos, insumos.

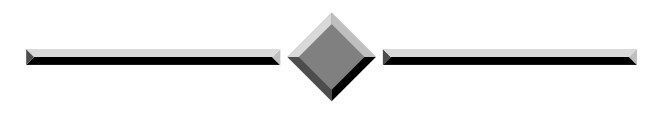

1 Recibido: 18 de noviembre, 2011. Aceptado: 12 de marzo, 2012. Este trabajo fue producto del proyecto de investigación, clave: UAEM/2534/2007U, financiado por la Universidad Autónoma del Estado de México, Toluca, Estado de México. El autor principal de este manuscrito fue co-responsable del proyecto.

2 Centro Universitario UAEM Temascaltepec-Universidad Autónoma del Estado de México. Km 67,5. Carretera Toluca-Tejupilco. Barrio de San Antonio S/N. C. P. 51300. Temascaltepec, Estado de México. samrere@hotmail.com. 2Posgrado en Administración. Instituto Tecnológico de Celaya, Guanajuato, México.samrere@hotmail.com (*Autor responsable); jh_martinez1214@yahoo.com.mx; dr_rojo70@ yahoo.com.mx; eugenio@itc.mx 


\section{INTRODUCCIÓN}

En el año 2009 la producción caprina en el mundo, se localizó principalmente en Asia y África, el primero lo representaron China, India, Pakistán y Bangladesh; y el segundo Nigeria, Sudán, Etiopía, Kenia y Somalia. En el continente americano destacaron Brasil, México, Argentina y Estados Unidos. En el mismo año, para México, del inventario total de especies domésticas, los caprinos ocuparon el tercer lugar después de bovinos y cerdos, con 8,9 millones de cabezas. Se sacrificaron aproximadamente $2,8 \mathrm{mi}-$ llones de caprinos en todo el país, equivalente a $43 \mathrm{mil}$ $t$ de carne en canal (FAO 2011).

En ese año, los principales compradores de carne caprina fueron Yemen, Arabia Saudita, Emiratos Árabes Unidos, en tanto que México, se ubicó en una posición baja y cercana a las 16,7 mil toneladas métricas; y los vendedores fueron Somalia, Omán e Irán y en una posición menor China (14 mil t) (FAO 2011).

En 2009, la producción mexicana de esta especie, se centró en los estados de Puebla $(16,1 \%)$, Oaxaca $(13,2 \%)$, Guerrero $(7,6 \%)$, Coahuila $(7,3 \%)$ y San Luis Potosí (6,8\%), quienes aportaron más del 50\% a la producción total (SIAP 2011). En el mismo año, el Estado de México produjo 1046 t de carne caprina, distribuidas en sus ocho Distritos de Desarrollo Rural (DDR); sin embargo, aproximadamente 59\% de la producción estatal se concentró en los DDR de Tejupilco y Coatepec Harinas, ubicados al suroeste de la Entidad, región eminentemente rural.

El Distrito de Desarrollo Rural (DDR) 076, con sede en Tejupilco, y el más importante en cuanto a producción de cabras, comprende los municipios de Temascaltepec, San Simón de Guerrero, Tejupilco, Luvianos, Amatepec y Tlatlaya. En ese año, solo este Distrito aportó 32,6\% al total estatal en producción de carne de cabra, proveniente de 6842 animales sacrificados (SIAP 2011). Dadas las condiciones climáticas, recursos forrajeros regionales, adaptación de la especie a condiciones adversas y manejo en general; la cabra, como especie de interés zootécnico, ha resultado ser una buena opción de producción animal para productores rurales de la zona sur del Estado de México.

Es importante analizar qué están haciendo los productores de cabras de la región para mantenerse en el sistema. Situación que condujo a formularse las siguientes preguntas: ¿cuánto gasta y percibe un productor, en promedio, para producir un animal desde la gestación hasta que se logra el peso adecuado para la venta al mercado, en el sistema de producción extensivo?; ¿qué otros factores de tipo económico determinan la permanencia de la actividad bajo el sistema mencionado? La hipótesis principal de este trabajo supone que el costo de producción representa menos que la mitad con relación al ingreso total por cabeza y la ganancia por unidad vendida supera al costo de producción.

El objetivo de este trabajo fue determinar los costos de producción y la rentabilidad de caprinos en un sistema de producción extensivo.

\section{MATERIALES Y MÉTODOS}

La investigación se realizó durante los meses de enero a junio de 2011 (periodo de estiaje) en dos comunidades que se destacaron por su producción de cabras en el municipio de Tejupilco, Estado de México: Monte de Dios y San Lucas del Maíz. La información provino de una encuesta estructurada y aplicada a cada productor seleccionado, de tal manera que si uno de ellos no deseaba participar de la encuesta, se optó por localizar al siguiente, pero cumpliendo con la muestra.

El instrumento incluyó variables socioeconómicas como: animales por productor, años en la actividad, escolaridad y miembros en la familia, percepción de ingresos por mes, otras actividades que complementan el ingreso familiar, pagos por utilizar agostaderos; etc., así como conceptos inherentes al costo de producción. El proceso inició desde la gestación hasta que el macho o hembra alcanzó 35 kilogramos $(\mathrm{kg})$ de peso o seis meses de edad, que corresponde al momento en que el productor generalmente decide venderlo (Rebollar et al. 2007). Posteriormente, se realizó tanto la caracterización del sistema de producción, como conceptos inherentes al costo de producir una unidad de producto hasta la venta del mismo y, cuantificación de costos mediante estadísticos de tendencia central y de dispersión (media y desviación estándar). Para el costo total de producción, se utilizaron conceptos microeconomómicos (Rebollar 2011, Parkin y Esquivel 2001, Varian 2006) como costos fijos, variables y totales de producción; así como conceptos de ganancia y ganancia económica. Esta última como diferencia entre Ingresos Totales (IT) y Costos Privados (costos explícitos e implícitos) (Rodríguez et al. 2008).

El concepto de ganancia en sí mismo, es la diferencia entre Ingreso Total (IT) y Costo Total 
(CT) (Rebollar 2011, Parkin 2001). Derivaciones adicionales de la Teoría de Costos fueron necesarios para estimar los costos y los ingresos a nivel de unidad o por cabeza y fueron: costo variable (CVMe) y fijo medio (CFMe), así como el costo medio total (CMeT) o total por unidad producida. Todos los cálculos se consideraron para estimar la rentabilidad por unidad producida en el sistema, para lo que se utilizó un tipo de cambio promedio a junio de 2011, de 11.8060 pesos por dólar estadounidense (BANXICO 2011).

\section{RESULTADOS Y DISCUSIÓN}

Con relación a características agroecológicas de la región de estudio, existió evidencia que permitió afirmar que se trata de un sistema de producción extensivo bien definido y orientado a la producción de animales Criollo X Nubia, específicamente para carne, cuyo principal destino final fue la elaboración del platillo típico denominado birria y tacos de chivo (Rebollar 2011; Rebollar et al. 2008). Al respecto, Arias et al. (2011) en su investigación sobre cabras criollas en la Mixteca oaxaqueña (México) menciona que estos animales se utilizan para fiestas populares, religiosas y de autoconsumo en forma de birria. Asimismo, Valerio (2009) afirmó que el carácter extensivo y tradicional de estas explotaciones ganaderas, dificulta la introducción al proceso productivo de razas mejoradas, pues los requerimientos nutricionales y de manejo, superan a los de razas Criollas existentes.

Tal sistema se encuentra determinado por características ecológicas predominantes de la región; esto es, precipitación adecuada, buena disponibilidad de pastos nativos con dominancia de arbustivas, herbáceas y gramíneas, así como esquilmos de temporal, provenientes de cosechas de maíz grano y asociación maíz-frijol, principalmente; lo que genera mejores oportunidades de alimentación estacional para los caprinos.

Durante la estación previa al estiaje, los rebaños tienen que disponer de un pastor, que generalmente son niños (mayores de seis años), amas de casa, mujeres jóvenes o personas de la tercera edad (Arias et al. 2011), para evitar que dichos rebaños causen daño a algunos cultivos como maíz (Zeas mays L.) y frijol (Phaseolus vulgaris L.) propiedad de los productores.

En el periodo de fin de lluvias y en primavera, muchos rebaños, en forma libre, pastorean y ramonean durante el día, generalmente entre las 08:00 $\mathrm{h}$ de la mañana hasta las 18:00 h de la tarde; similar a lo encontrado por Arias et al. (2011); después, a los animales se les encierra en corrales rústicos del productor, lo que concuerda con Valerio (2009), Valerio et al. (2009), Bellido et al. (2001) y Hernández et al. (2011). La cercanía de los corrales a la vivienda del productor es variada, pero en más de la mitad de los encuestados, se encontró que tales instalaciones se localizaron cerca de su hogar. En ocasiones, se aprovecha la sombra de árboles silvestres y solo se construye un techo pequeño para cubrirlos de lluvias en esa época, pues la cabra, normalmente, le teme al agua (Lesur 2004), en consonancia con Bedotti et al. (2007).

Con relación al análisis socioeconómico, la edad de los productores varió en 56,0 \pm 5,0 años, en concordancia, Trujillo et al. (2010) en su diagnóstico de cabras realizado en Tecamachalco, Puebla, México, mencionaron 53,18 años como edad promedio de los productores; el nivel de escolaridad en 4,0 \pm 1,5 años, los miembros por familia en 5,0 $\pm 2,0$, similar a lo encontrado por Hernández et al. (2011) y Trujillo et al. (2010); en tanto que el ingreso mensual, producto de actividades externas a la producción de caprinos, en US\$186,3 \pm 93,2 (estimado y con base en información proveniente del productor); el promedio de cabezas de éste se encontró en 14,0 \pm 5,0. Arias et al. (2011) mencionaron que los criadores de cabras en un municipio de la Mixteca Baja Oaxaqueña percibieron, en promedio, 227 US\$ por mes como ingreso de fuentes relacionadas a la actividad caprina.

Con relación a la tenencia de cabras, Arias et al. (2011) encontraron que el promedio de animales por productor en la Mixteca oaxaqueña fue 37; Trujillo et al. (2010), en su investigación sobre cabras Criollas x Nubia, en Tecamachalco, Puebla, México, mencionaron que el promedio de animales por productor fue 16 \pm 2 caprinos; asimismo, García, et al (1994), en un estudio sobre caracterización de sistemas de producción caprina en Tejupilco, Estado de México, concluyeron que del total de animales que dispuso el productor, $52,2 \%$ (dieciséis animales) correspondió a cabras; similar a lo encontrado por Bedotti et al. (2007) en el oeste pampeano Argentino y Valerio et al. (2009) para una región de República Dominicana.

En un trabajo sobre caprinos en Torreón, Coahuila, Cantú et al. (2004), afirmaron que $50 \%$ de los productores tenían entre 10 y 40 cabezas, 28\% de 41-80 cabezas y $22 \%$ de $81-120$ cabezas por productor. Por 
su parte, Hernández et al. (2004), encontraron que el número de cabras por productor en la Mixteca poblana varió de 30 hasta 110 cabezas, concentrándose de 60 a 90 caprinos en rebaños de la mayoría de los productores; similar a lo encontrado por Valerio et al. (2009), en una región de República Dominicana.

Las familias de criadores de caprinos se caracterizan por tener un jefe de edad avanzada y un gran número de miembros; así la edad del jefe de familia fue $51,8 \pm 13,8$ años y el promedio de escolaridad 3,2 \pm 4,2 años; sin embargo, sólo 41,3\% de los encuestados tuvieron educación primaria (Castillo y García 2001).

Del total de criadores de caprinos en la región de estudio, 97\% habitan en vivienda propia, construida generalmente de adobe, pisos de cemento (a veces de tierra "apelmazada"), con dos habitaciones, cocina y "corredor" en promedio y fosa séptica; los techos de sus casas están provistos de teja y de lámina de asbesto y con pocas ventanas; mientras que el resto utilizan casas prestadas, o en el último de los casos, rentadas. En tenencia de la tierra, dominó la propiedad privada (99\%), en tanto que en algunas comunidades, aún existen remanentes de ejido o propiedad comunal con la garantía, de parte de los tenedores, de poder transferir, heredar o vender su propiedad.

En la región de estudio, la comercialización de caprinos, no constituye un problema para los productores (Rebollar 2011, Rebollar et al. 2007), ya que todo lo que se oferta es adquirido inmediatamente (relacionado a estos animales); es decir, generalmente basta con que el propietario del rebaño desee vender alguno o varios de sus animales y la venta se realiza sin dificultad alguna (naturalmente, no en ese instante). Incluso, en gran parte de la región, los propietarios de animales no requieren viajar a la plaza principal a ofrecer sus animales en venta, sino que el introductor de ganado o compradores ocasionales, acude o acuden con su medio de transporte hasta la vivienda del productor para realizar la operación.

Como se dijo, no es común que acordada la negociación, tanto el comprador como el vendedor, pesen al animal en báscula, todo es a través de compra directa. Para la región de estudio, el rendimiento en canal (de peso vivo a peso en canal) se ubicó en $51 \%$, con un precio promedio de la canal caliente (en el mercado) de US\$ 6,8/kg (precio vigente hasta junio de 2011 en el Rastro Municipal de Tejupilco, Estado de México). Así, un animal de $35 \mathrm{~kg}$ de peso vivo, en promedio rinde $18 \mathrm{~kg}$ en canal (sin cocer) y $9,7 \mathrm{~kg}$ con cocción (Rebollar et al. 2008).

\section{Alimentación}

En el sistema extensivo, la alimentación se basa principalmente en el ramoneo y pastoreo en agostaderos (Rebollar y Rojo, 2010), poca o nula suplementación $(80 \%$ de productores encuestados no suplementa algún nutriente), prácticas de desparasitación (100\% de los productores desparasitan a sus animales; de éstos, $80 \%$ lo realizan según la edad del animal, o al inicio de cada estación) y se les encierra por las noches en instalaciones rústicas propiedad de productores, en convivencia con especies pecuarias menores como aves de corral (gallinas y guajolotes), en consonancia con Bedotti et al. (2007), Valerio (2009).

La producción de caprinos utiliza terrenos menos productivos (aunque fértiles), generalmente no aptos para actividades agrícolas, que ocupan la mayor parte de la zona de estudio, con pendientes, en algunas ocasiones, mayores a $45 \%$. Generalmente la caprinocultura no dispone de otras fuentes de alimentación durante el año, es por ello que requieren de grandes extensiones de terreno. De hecho, más del 50\% de los productores encuestados destinan poco más de cuatro hectáreas para el pastoreo de sus animales.

En este sistema (extensivo de producción), predomina la baja tecnificación y se practica el sobrepastoreo, como lo afirman Valerio (2009), Valerio (2010) y Bedotti et al. (2007), donde los caprinos aprovechan la vegetación nativa de la región. En partes altas de la región (desde el punto de vista orográfico), se encuentran ecosistemas pino-encino y en las áreas más cálidas pastos, hierbas arbustivas, leguminosas, propias de la selva baja caducifolia existentes en la región sureña de la Entidad, que son las que prefieren las cabras. Las especies más valiosas para las cabras en la zona de estudio, por su palatabilidad son arbustivas como: Leucaena sp. (Guaje), Pithecellobium dulce (Guamúchil), Acacia farnesiana (Huizache), Guazuma ulmifolia (Guázima), Prosopis juliflora (Mezquite), Quercus sp. (Encino) (Camacho et al. 2010).

Entre los pastos y herbáceas importantes se tienen: Mulhembergia tenuifloia (Zacatón), Buchloe dactyloides, Hilaria cenchroides, Lycurus phleoides (Cola de zorra), Sporolobus sp., Bouteloua gracilis, Dalea sp. (Engorda cabras), Festuca sp. Teniendo la 
oportunidad de ser selectiva, la cabra va a pastorear o ramonear todo tipo de plantas, prácticamente come de todo (Camacho et al. 2010).

La suplementación no es frecuente, y ocasionalmente su práctica sólo ocurre en la época del estiaje $\mathrm{y}$, esta se realiza a base de esquilmos agrícolas (que generalmente no son utilizados por el productor en la alimentación de especies mayores, como bovinos), similar a lo encontrado por Valerio (2009), Bedotti et al. (2007) y Hernández et al. (2011).

Algunos productores $(<20 \%)$ suplementan con tequezquite, del cual no se conoce su composición ni contenido de algunas posibles sustancias que, incluso, podrían ser nocivas para la salud de animales; otros productores utilizan sal común o sal de cocina. Incluso, bajo el sistema extensivo no es común que los productores asignen sales minerales, pues la alimentación natural de cabras, bajo el pastoreo, les genera esa ventaja, dado que gran parte de los minerales la obtienen del consumo de distintas plantas, arbustos u hojas de árboles que consumen durante el día (Rebollar y Rojo 2010, Lesur 2004).

\section{Costos de producción}

En el sentido estricto, el costo de producción, bajo condiciones extensivas de producción de cabras, debe considerar el lapso desde que la hembra queda preñada, nacimiento, destete, crecimiento, desarrollo y venta del animal, con un peso vivo superior a $30 \mathrm{~kg}$.

No obstante, debido a que en este sistema de producción, no existe control de rebaños, y que el macho todo el tiempo convive con las hembras en agostaderos, como le mencionó Bedotti et al. (2007), Valerio et al. 2010, en este trabajo se omite, como costos variables, el costo de preñez, alimentación de la madre durante la gestación (150 días) y del cabrito del nacimiento al destete (60 días), alimentación predestete, agua de abrevaderos, sales minerales, vitaminas, desparasitantes externos y costos por flete. Como costos fijos, el trabajo del pastor, renta de potreros y cuotas de asociación. Tales conceptos, se convierten en un costo constante cuyo valor es nada.

Dentro de la contabilidad total, es claro que los rubros antes señalados deberían incorporarse; de hacerlo, el sistema de producción no lograría sobrevivir, de no ser por la contribución de la economía familiar, la cual aporta insumos y recursos con costos
Cuadro 1. Costos de producción en caprinos bajo el sistema extensivo, 2011, Sur del Estado de México, México.

\begin{tabular}{lrr}
\hline Concepto & $\begin{array}{r}\text { Costo } \text { (US\$ } \\
\text { por cabeza) }\end{array}$ & \multicolumn{1}{l}{$\%$} \\
\hline Costo variable & $\mathbf{3 , 8}$ & $\mathbf{4 3 , 7}$ \\
Desparasitantes & & \\
$\quad$ Internos & $1,8 \pm 0,3$ & 20,7 \\
Jeringas & $0,3 \pm 0,1$ & 3,5 \\
Costo por inyectar & $1,7 \pm 0,4$ & 19,5 \\
\hline Costo fijo & $\mathbf{4 , 9}$ & $\mathbf{5 6 , 3}$ \\
Corrales de encierro nocturno** & 1,1 & 12,5 \\
Sombreadero & 1,4 & 16,0 \\
Bebederos & 0,07 & 0,7 \\
Saladeros & 0,07 & 0,7 \\
Mantenimiento de corrales & 2,3 & 26,4 \\
\hline Costo total & $\mathbf{8 , 7}$ & $\mathbf{1 0 0 , 0}$ \\
\hline
\end{tabular}

Nota: el gasto de corrales fue de US\$ 84,7, del sombreadero en US $\$ 105,9$, los saladeros de US $\$ 5,3$ y los bebederos US $\$ 8,5$ en promedio. 2008. Los beberos y saladeros tienen una vida útil, estimada, de cinco años.

** US\$ 84,7/5 años de vida útil del activo = US\$ 16,9 por año; US\$ $16,9 / 12$ meses $=$ US $\$ 1,4$ por mes; US\$ $1,4 / 14 \pm$ cinco animales promedio $=$ US $\$ 0,1$ por cabeza/mes; US $\$ 0,1 / 30$ días $=$ US\$ 0,0034/día, luego, al multiplicarse por 330 días = US\$ 1,1 por cabeza.

de oportunidad equivalentes a cero (no hay otra mejor alternativa), tales como mano de obra familiar (Valerio et al. 2009), agua de abrevaderos, utilización tanto del agostadero como del alimento (forraje, hierbas, plantas diversas, etc.), inseminación natural (monta natural); que son rubros de costos considerados como erogaciones hundidas o erogaciones sepultadas (Parkin y Esquivel 2001). Otros desembolsos no contabilizados, debido a que el productor no los incurrió, fueron: energía eléctrica en corrales, seguro animal, inseminación artificial, asistencia técnica.

Por tanto, desde la gestación a la venta de un caprino (35 kg de peso) transcurren, en promedio, 330 días, distribuidos en: 150 días de gestación, más 180 días desde el nacimiento hasta que pesan $35 \mathrm{~kg}$ en pie (Hernández et al. 2011). Así, bajo las condiciones 
planteadas, el costo total de producción por cabeza se ubicó en US\$ 8,7, de este, el costo variable fue US\$ 3,8 y el fijo de US\$ 4,9; lo que concuerda con Valerio (2009). El componente principal del costo variable fue el concepto desparasitantes y del fijo el mantenimiento de corrales (Cuadro 1). Solo para este caso, el costo fijo fue superior al variable, primero, debido a lo intrínseco del sistema, segundo, por el gasto en instalaciones.

Si bien, con fundamento en la Teoría Económica, de que los costos fijos no dependen de la producción, ni del mercado y deben asumirse aún en ausencia de producción (Valerio 2009, Acero et al. 2004), por ello se consideró que el activo fijo tiene una vida útil y se vuelve obsoleto (características inherentes a todo activo fijo), al final de la cual debe realizarse otro desembolso para su reposición.

Para la estimación del ingreso total (IT) por cabeza, la mayoría de los productores venden sus animales con un peso superior a los $30 \mathrm{~kg}$; sin embargo, lo anterior podría variar, porque está en función del momento en que ocurra la venta y del tipo de animal que prefiera el comprador, para lo cual se consideró un peso promedio a la venta de $35 \mathrm{~kg}$ y un precio por kilogramo de 2,033 dólares; con lo que el IT $=($ US\$ 2,033) $(35$ $\mathrm{kg})=\mathrm{US} \$ 71,2$.

Con ello, la ganancia neta por animal fue US\$ 62,5, similar a lo encontrado por Hernández et al. (2011), Valerio (2009), Valerio (2010) y Jeannite (2011); en tanto que la relación beneficio-costo se ubicó en 8,1 , lo que indicó que por cada dólar invertido el productor habría percibido (bajo el supuesto de que realizó la transacción), US\$ 7,1 (Cuadro 2).

En las condiciones planteadas, el costo de producción de cabras, bajo el sistema extensivo, fue menos que la tercera parte del ingreso por venta, debido a la poca magnitud de gasto que realizan los productores.

Cuadro 2. Comparación del costo de producción y la ganancia por venta de caprinos, bajo el sistema extensivo. Sur del Estado de México, México. 2011.

\begin{tabular}{cccc}
\hline $\begin{array}{c}\text { Costo (US\$/ } \\
\left.\mathrm{cab}^{*}\right)\end{array}$ & $\begin{array}{c}\text { Ingreso Total } \\
\left(\mathrm{US} \$ \mathrm{cab}^{*}\right)\end{array}$ & $\begin{array}{c}\text { Ganancia } \\
\left(\mathrm{US} \$ / \mathrm{cab}^{*}\right)\end{array}$ & $\mathrm{RB} / \mathrm{C}^{* *}$ \\
\hline 8,7 & 71,2 & 62,5 & 8,1 \\
\hline
\end{tabular}

* US\$/cab: dólares estadounidenses por cabeza. ** RB/C: Relación Beneficio-Costo.

ISSN: 1021-7444
En consecuencia, la ganancia por unidad vendida fue siete veces superior al costo de producción. Del costo total, el fijo fue mayor al variable, situación que no necesariamente debe ser similar a otros sistemas de producción de cabras, pues durante el periodo de estudio, los productores desembolsaron más recursos en instalaciones que en la mejora de sus propios rebaños.

\section{LITERATURA CITADA}

Acero, R; García, A; Ceular, N; Artacho, C; Martos, J. 2004. Aproximación metodológica a la determinación de costes en la empresa ganadera. Archivos de Zootecnia 53:91-94.

Arias, L; Soriano, R; Sánchez, E; González, C; Rivera, L. 2011. Características técnicas y socioeconómicas de los sistemas de producción caprina en un municipio de la Mixteca Baja oaxaqueña. p. 335-345. In La ganadería ante el agotamiento de los paradigmas dominantes, Vol. 2. Coordinadores: Beatriz A. Cavalloti Vázquez, Benito Ramírez Valverde, Francisco Ernesto Martínez Castañeda, Carlos Felino Marcof Álvarez, Alfredo Cesín Vargas. Universidad Autónoma Chapingo, Chapingo, Estado de México. 424 p.

BANXICO (Banco de México). 2011. Tipo de cambio para solventar obligaciones en moneda extranjera. En línea. Consultado el 2 de julio de 2011. Disponible en: http:// www.banxico.org.mx/SieInternet/consultarDirectorioInternetAction. do? accion=consultarCuadro\&idCuadr $\mathrm{o}=\mathrm{CF} 86 \&$ sector $=6 \&$ locale $=\mathrm{es} \#$

Bedotti, D; Gómez, AG; García, A; Sánchez, M; Perea, J; Rodríguez, V. 2007. Estructura productiva de las explotaciones caprinas del oeste pampeano (Argentina). Archivos de Zootecnia 56(213):91-94.

Bellido, M; Escribano, S; Mesías, D; Rodríguez, L; Pulido, G. 2001. Sistemas extensivos de producción animal. Archivos de Zootecnia 50:465-489.

Camacho, LM; Rojo, R; Salem, A; Provenza, D; Mendoza, D; Avilés, F; Montañez, OD. 2010. Effect of season on chemicals composition and in si tu degradability in cows and in adapted and anadapted goats of three Mexican browse species. Animal Feed Science and Technology 155:206-212.

Cantú, JE; Acevedo, C; Ríos, S. 2004. Diagnóstico situacional de la caprinocultura en tres comunidades de Jimulco. Municipio de Torreón, Coahuila. Memorias. XIX Reunión Nacional sobre Caprinocultura. Acapulco, Gro. Octubre de 2004. p. 403-406. 
Castillo, J; García, O. 2001. Las zonas secas, los sistemas de producción y el mejoramiento genético en el contexto de la ganadería caprina y sus perspectivas en Venezuela. XVI Reunión Nacional sobre Caprinocultura. Octubre 17-19. Veracruz, Ver. México. p. 420-424.

FAO (Food and Agriculture Organization of the United Nations). 2011. FAOSTAT. Estadísticas (en línea). Consultado el 21 de enero de 2011. Disponible en http:// faostat.fao.org/site/573/default.aspx\#ancor

García, T; García, A; de Lucas, J. 1994. Caracterización de los sistemas de producción de ganado caprino en el sur del Estado de México (municipio de Tejupilco). Memorias. I Congreso Internacional y II Nacional de investigación en sistemas de producción agropecuaria. CICA-UAEM. Toluca, Estado de México. p. 284-293.

Hernández, JE; Franco, FJ; Pedraza, R. 2004. Productores $\mathrm{y}$ hatos caprinos que caracterizan socialmente un sistema de producción en la Mixteca poblana (Piaxtla). XIX Reunión Nacional sobre Caprinocultura. Memoria. Acapulco, Guerrero. Colegio Superior Agropecuario del estado de Guerrero. p. 407-410.

Hernández, JE; Franco, FJ; Villarreal, A; Camacho, C; Pedraza, M. 2011. Caracterización socioeconómica y productiva de unidades caprinas familiares en la mixteca poblana. Archivos de Zootecnia 60(230): 175-182.

Jeannite, A. 2011. Estudio de la viabilidad de la ganadería caprina en el noroeste de la República Dominicana. Trabajo de fin de Máster. Universidad de Córdoba, España. 23 p. (en línea). Consultado 13 marzo 2012. Disponible en http://www.uco.es/zootecniaygestion/ img/pictorex/01_10_00_Jeannite_Amos.pdf

Lesur, L. 2004. Manual del ganado caprino. Una guía paso a paso. Editorial Trillas. México, D. F. 80 p.

Parkin, M; Equivel, G. 2001. Microeconomía. Versión para América Latina. Quinta Edición. Editorial Addison Wesley. Naucalpan de Juárez, Estado de México. México. 541 p.

Rebollar, S; Hernández, J; García, JA; García, R; Torres, G; Bórquez, JL; Mejía, P. 2007. Canales y márgenes de comercialización de caprinos en Tejupilco y Amatepec, Estado de México. Agrociencia 41(3):363-370.

Rebollar, S; Hernández, J; González, FJ; Rojo, R; Cardoso, D, Albarrán, B; García, A. 2008. Elaboración y costos de producción de la birria de chivo en el sur del Estado de México. p. 334-340. In Beatriz A. Cavallotti Vázquez, Benito Ramírez Valverde y Carlos F. Marcof Álvarez. eds. Ganadería y Desarrollo Rural en Tiempo de Crisis. Primera Edición. Universidad Autónoma Chapingo. Chapingo, Estado de México. 356 p.

Rebollar, S; Rojo, R. 2010. El Estado del arte de la caprinocultura en el sur del Estado de México. Universidad Autónoma del Estado de México. 180 p.

Rebollar, S. 2011. Métodos para estimar costos de producción y rentabilidad. Experiencias y casos de estudio. Editorial Académica Española. 103 p.

Rodríguez, V; Bao, R; Cárdenas, L. 2008. Formulación y evaluación de proyectos de inversión. Ed. Limusa. México. D. F. 454 p.

Trujillo, CX; Vargas, S; Torres, G; Bustamante, A; Becerril, CM; Guerrero, JD. 2010. Análisis del proceso de introducción de cabras exóticas en los sistemas campesinos de la Mixteca poblana. p. 425-433. In Los grandes retos para la ganadería: Hambre, Pobreza y Crisis ambiental. Coordinadores: Beatriz A. Cavalloti Vázquez, Carlos F. Marcof Álvarez y Benito Ramírez Valverde. Primera Edición. Universidad Autónoma Chapingo. Chapingo, Estado de México. 433 p.

SIAP (Servicio de Información Agroalimentaria y Pesquera). 2011. Resumen de la población ganadera en México. (en línea). Consultado 21 enero 2011. Disponible en http://www.siap.gob.mx/index.php?option=com_cont ent $\&$ view $=$ article $\& i d=3 \&$ Itemid $=29$.

Valerio, DJ. 2009. Análisis de competitividad del sistema ovino y caprino del noroeste de la República Dominicana. Tesis Doctoral. Universidad de Córdoba, España. 246 p.

Valerio, D; García, A; Perea, J; Acero, R; Gómez, G. 2009a. Caracterización social y comercial de los sistemas ovinos y caprinos de la región noroeste de la República. Interciencia 34(9):637-644.

Valerio, D; García, A; Acero, R; Perea, J; Tapia, M; Romero, M. 2010. Caracterización estructural del sistema ovino-caprino de la región noroeste de República Dominicana. Archivos de Zootecnia 59(227):333-343.

Varian, R. 2006. Microeconomía intermedia. 7 ed. Editorial Antoni Bosch. Barcelona, España. 789 p. 